\title{
All-Optical Stochastic Logic Gate Based on a VCSEL With Tunable Optical Injection
}

\author{
Matias F. Salvide, Cristina Masoller, and Maria Susana Torre
}

\begin{abstract}
We study the dynamics of a vertical-cavity surfaceemitting laser with continuous-wave orthogonal optical injection from a tunable laser. We use the dynamical properties of polarization bistability and the interplay with internal or external noise to demonstrate numerically a reliable logic output to two logic inputs encoded in the optical frequency of the injected light. This all-optical configuration is more than ten times faster than the electro-optical implementation and has the advantage of operating at constant injection power.
\end{abstract}

Index Terms-Vertical-cavity surface-emitting lasers (VCSELs), semiconductor lasers, laser dynamics, polarization bistability, polarization switching, optical injection, noise.

\section{INTRODUCTION}

O PTICAL injection is commonly employed to achieve optical bistability and a lot of research has been devoted to exploit the response of optical bistable systems for alloptical signal processing, optical switching, and optical storage. Vertical-cavity surface-emitting lasers (VCSELs) are good candidates for optical signal processing in photonic networks [1], [2] due to their many advantages over edge-emitting devices [3], including polarization switching and bistability.

Polarization switching and bistability can occur in freerunning VCSELs, and can also be induced by external optical injection [4]-[10]. Multi-transverse-mode emission is also observed in free-running VCSELs, and in this situation, external optical injection can be used for polarization and/or transverse mode selection [11]-[16].

Two types of optical injection schemes have been considered in the literature, one in which the polarization of the injected light is the same as that of the free-running laser (this scheme has been referred to as coherent injection), and another in which the polarization of the injected light is orthogonal to that of the free-running laser (referred to as orthogonal injection). With orthogonal injection polarization bistability can be induced by varying the detuning of the injected light, or by varying the injected power [17]-[29].

Manuscript received May 29, 2013; revised July 5, 2013; accepted July 24, 2013. Date of publication August 1, 2013; date of current version September 16, 2013. This work was supported by CONICET under Grant PIP-114200801-00163, rgentina. The work of C. Masoller was supported in part by EOARD under Grant FA8655-12-1-2140, in part by the Spanish MCI under Grant FIS2012-37655-C02-01, in part by the Generalitat de Catalunya under Grant 2009 SGR 1168, and in part by the ICREA Academia program.

M. F. Salvide and M. S. Torre are with the Instituto de Física "Arroyo Seco", UNCPBA, Tandil 7000, Argentina (e-mail: marita@exa.unicen.edu.ar; msalvide@exa.unicen.edu.ar).

C. Masoller is with the Department de Fisica i Enginyeria Nuclear, Universitat Politecnica de Catalunya, Terrassa 08222, Spain (e-mail: cristina.masoller@upc.edu).

Color versions of one or more of the figures in this paper are available online at http://ieeexplore.ieee.org.

Digital Object Identifier 10.1109/JQE.2013.2276122
In this work we demonstrate that the polarization properties of a VCSEL with orthogonal injection can be exploited to induce switchings between the two linear polarizations, $x$ and $y$, in response to changes in the optical frequency of the injected light, giving a reliable logic response to two logic inputs that are encoded in the frequency variation. The output response is interpreted as 0 if the laser emits the free-running polarization and as 1 if emits the orthogonal one. We show that due to the nontrivial interplay of orthogonal injection and polarization bistability, with an adequate level of noise the VCSEL can give the correct logic response to the logic inputs.

This phenomenon (which has been referred to as stochastic logic resonance [30], [31]) has been demonstrated in Refs. [32], [33] using different setups: in [32], a free-running VCSEL, with the logic inputs encoded in a modulation of the pump current, and in [33], a VCSEL with coherent optical injection, with the logic inputs encoded in a modulation of the injected power. We compare the performance of these encoding schemes with the one proposed here using orthogonal injection and show that with orthogonal injection the logic gate operates more than ten times faster than in [32] and as fast as in [33]. The implementation demonstrated here has the advantage that requires a simpler setup than in [33], as it requires $\mathrm{cw}$ injection power from a tunable master laser.

This paper is organized as follows. Section II presents the model used for describing the VCSEL polarization dynamics under orthogonal injection. Section III presents the encoding scheme, by which two logics inputs are encoded in three values of the detuning of the injected light, which has a polarization that is orthogonal to that of the free-running laser, and the logic output is decoded from the polarization of the light emitted by the laser. Section IV presents the results. We evaluate the reliability of the logic output response as a function of the VCSEL parameters and as a function of the optical injection parameters. We consider two injection scenarios: i) the polarization of the free-running laser is the lowfrequency one $(x)$ and the orthogonal injection induces a switching to the high-frequency polarization $(y)$; ii) the polarization of the free-running laser is the high-frequency polarization $(y)$ and the orthogonal injection induces a switching to the low-frequency polarization $(x)$. We show that these two scenarios display similar features in terms of the model parameters required for the reliable operation: in both cases the minimum bit time for successful operation is about $2 \mathrm{~ns}$ and the minimum injection strength increases with the pump current. However, they have different noise sensitivity and require different injection strengths. We conclude in section $\mathrm{V}$ with a summary and a discussion of the results. 


\section{MODEL}

We use the well-know spin-flip model [34] for a VCSEL operating in the fundamental transverse mode, extended to account for external optical injection. The equations written in the frequency reference frame of the injected field are

$$
\begin{aligned}
\frac{d E_{x}}{d t}= & \kappa(1+i \alpha)\left(N E_{x}+i n E_{y}-E_{x}\right)-i\left(\gamma_{p}+\Delta \omega\right) E_{x} \\
& -\gamma_{a} E_{x}+\sqrt{\beta_{s p} \gamma_{N} N} \xi_{x}+\kappa_{x} E_{i n j} \\
\frac{d E_{y}}{d t}= & \kappa(1+i \alpha)\left(N E_{y}-i n E_{x}-E_{y}\right)+i\left(\gamma_{p}-\Delta \omega\right) E_{y} \\
& +\gamma_{a} E_{y}+\sqrt{\beta_{s p} \gamma_{N} N} \xi_{y}+\kappa_{y} E_{i n j} \\
\frac{d N}{d t}= & -\gamma_{N}\left[N\left(1+\left|E_{x}\right|^{2}+\left|E_{y}\right|^{2}\right)\right] \\
& +\gamma_{N} \mu-i \gamma_{N} n\left(E_{y} E_{x}^{*}-E_{x} E_{y}^{*}\right) \\
\frac{d n}{d t}= & -\gamma_{s} n-\gamma_{N} n\left(\left|E_{x}\right|^{2}+\left|E_{y}\right|^{2}\right) \\
& -i \gamma_{N} N\left(E_{y} E_{x}^{*}-E_{x} E_{y}^{*}\right) .
\end{aligned}
$$

Here $E_{x}$ and $E_{y}$ are the two orthogonal linearly polarized slowly varying components of the field and $N$ and $n$ are two carrier variables. $N$ accounts for the total population inversion between conduction and valence bands, while $n$ is the difference between the population inversions for the spin-up and spin-down radiation channels. The VCSEL parameters are: $\kappa$ is the field decay rate, $\gamma_{N}$ is the decay rate of $N, \gamma_{s}$ is the spin-flip relaxation rate, $\alpha$ is the linewidth enhancement factor, $\gamma_{a}$ is the linear dichroism, $\gamma_{p}$ is the linear birefringence and $\mu$ is the pump current parameter (normalized such that the solitary threshold is at $\mu=1$ in the absence of anisotropies). Spontaneous emission noise is taken into account by $\xi_{x}(t)$ and $\xi_{y}(t)$ that are independent complex Gaussian noise terms of zero mean and time correlation given by $<\xi_{i}(t) \xi_{j}^{*}\left(t^{\prime}\right)>=$ $2 \delta_{i j} \delta\left(t-t^{\prime}\right)$. We define the noise strength parameter as $D=$ $\gamma_{N} N \beta_{s p}$ with $\beta_{s p}$ being the spontaneous emission factor. We consider parameters well above threshold and approximate $N \approx 1$ since above threshold the $N$ is clamped to the threshold value.

The optical injection parameters are: $\kappa_{x}, \kappa_{y}, E_{i n j}$ and $\Delta \omega$; where $\kappa_{x}\left(\kappa_{y}\right)$ is the injection coefficient in the $x(y)$ polarization (is either 0 or $\kappa$ and is used to select the type of orthogonal injection as described below), $E_{i n j}$ is the injected field amplitude and $\Delta \omega=\omega_{i n j}-\omega_{r e f}$ is the detuning of the injected field, defined by the difference between the angular optical frequency of the injected field $\omega_{i n j}$, and the reference angular frequency defined as $\omega_{\text {ref }}=\left(\omega_{x}+\omega_{y}\right) / 2$, with $\omega_{x}$ and $\omega_{y}$ being the angular optical frequencies of the $x$ and $y$ polarizations of the free-running VCSEL, $\omega_{x}=-\gamma_{p}+\gamma_{a} \alpha$, $\omega_{y}=\gamma_{p}-\gamma_{a} \alpha$ [34].

We consider parameters such that the free-running VCSEL emits either the low-frequency, $x$ polarization, or the high frequency, $y$ polarization. This allows to consider two types of orthogonal injection: when the polarization of the freerunning laser is the high-frequency $y$ polarization we consider the injection of $x$ polarized light, in the following referred to as $x$-polarized injection; when the free-running laser emits the low-frequency $x$ polarization, the orthogonal injection is on the $y$ polarization and is referred to as y-polarized injection. Thus,

i) for $x$ polarized injection, $\kappa_{x}=\kappa$ and $\kappa_{y}=0$;

ii) for $y$ polarized injection, $\kappa_{y}=\kappa$ and $\kappa_{x}=0$;

where $\kappa$ is the field decay rate. Due to the interplay of birefringence (represented by the parameter $\gamma_{p}$ ) and the phaseamplitude coupling (represented by the $\alpha$ factor) these two types of orthogonal injection are not equivalent.

\section{INPUT-OUTPUT LOGIC ENCODING}

In our implementation of the stochastic logic gate using orthogonal injection, two logic inputs are encoded in three detuning values, $\Delta \omega_{\mathrm{I}}, \Delta \omega_{\mathrm{II}}$ and $\Delta \omega_{\mathrm{III}}$, of the injected field, and the output response is decoded from the polarization of the emitted light: the logic output is interpreted as 1 if the laser emits the free-running polarization and as 0 if it emits the orthogonal one. Therefore, when using $x$-polarized injection, $y$ represents 1 and $x$ represents 0 , while when using $y$-polarized injection, $x$ presents 1 , and $y$ represents 0 .

Then, the truth table of the fundamental logical operations OR, AND (and their negations, NAND and NOR) can be implemented. To fix the ideas, let us consider Table 1 for logic OR. We have four logic input sets: $(0,0),(0,1),(1,0)$, and $(1,1)$. Representing the $(0,1)$ and $(1,0)$ with the same detuning $\left(\Delta \omega_{\text {III }}\right)$ we can encode the four inputs with a three-level signal. For simplicity, $\Delta \omega_{\text {I }}$ and $\Delta \omega_{\text {III }}$ are defined symmetrically from $\Delta \omega_{\text {II. }}$. Then, the detuning variation, $\Delta \omega_{\mathrm{c}}$, is defined as

$$
\Delta \omega_{\mathrm{c}}=\Delta \omega_{\mathrm{II}}-\Delta \omega_{\mathrm{I}}=\Delta \omega_{\mathrm{III}}-\Delta \omega_{\mathrm{II}} .
$$

The three-level signal used to vary $\Delta \omega$ is such that is constant during a time interval $T_{1}$ and then is followed a fast ramp (up or down) $T_{2}$ to the detuning corresponding to the next bit. Thus, the duration of the bit, referred to as bit time, is $T=T_{1}+T_{2}$ with $T_{2}<T_{1}$ (unless otherwise noted, $\left.T_{2} / T_{1}=0.1\right)$.

To quantify the reliability of the logic gate we compute the probability of obtaining the correct logic output (referred to as success probability), using the same criteria as in [32], [33]. The idea is to determine the logic output ( 1 or 0 ) by measuring only the intensity emitted in one polarization. Specifically, we consider the free running polarization. To fix the ideas, let us consider $x$-polarized injection (such that the free-running laser emits the $y$-polarization). Then, we determine the logic output in terms of the percentage of light emitted in the $y$ polarization: when $y$ is the correct polarization, we consider that the laser response in a bit is correct if, during the bit, the percentage of light emitted in the $y$ polarization is above a certain value. In [32], [33] the following percentages were used: $90 \%$ or $80 \%$ or $70 \%$, to allow for a transient at the beginning of the bit during which the laser might have to switch the polarization.

When $y$ is not the correct output polarization, then, to consider that the laser output response is correct we require that no more than a certain percentage of light $(10 \%$, or $20 \%$ or $30 \%$ ) is emitted in the wrong $y$ polarization.

Therefore, if we use the most restrictive criterion (referred to as 90/10), the laser output response is correct if: 
TABLE I

INPUT - OUTPUT COMBINATION FOR AN OR GATE: The INPUTS ARE

ENCODED IN THE DETUNING OF THE INJECTED Light AND THE OutPut is Decoded From the Polarization of THE LIGHT EMITTED BY THE VCSEL

\begin{tabular}{|c|c|c|c|c|c|}
\hline \multicolumn{2}{|c|}{ OR Logic Gate } & \multicolumn{2}{c|}{ Emitted polarization } & \multirow{2}{*}{ Detuning } \\
\hline \multicolumn{2}{|c|}{ Inputs } & Output & $\begin{array}{c}\text { X-polarized } \\
\text { injection }\end{array}$ & $\begin{array}{c}\text { Y-polarized } \\
\text { injection }\end{array}$ & \\
\hline 1 & 1 & 1 & $\mathrm{Y}$ & $\mathrm{X}$ & $\Delta \omega_{\mathrm{I}}$ \\
\hline 1 & 0 & 1 & $\mathrm{Y}$ & $\mathrm{X}$ & $\Delta \omega_{\mathrm{III}}$ \\
\hline 0 & 1 & 1 & $\mathrm{Y}$ & $\mathrm{X}$ & $\Delta \omega_{\mathrm{III}}$ \\
\hline 0 & 0 & 0 & $\mathrm{X}$ & $\mathrm{Y}$ & $\Delta \omega_{\mathrm{II}}$ \\
\hline
\end{tabular}

i) $y$ is the correct polarization, more than $90 \%$ of the light emitted during the bit is emitted in the $y$ polarization.

ii) $y$ is not the correct polarization, less than $10 \%$ of the light emitted during the bit is emitted in the $y$ polarization.

The more permissive criterion (70/30) allows for up to $30 \%$ of the light to be emitted in the wrong polarization, and requires a minimum of $70 \%$ in the correct one.

\section{Results}

In this section we present the results of the simulations of the model equations. The Platen explicit order 1.5 strong scheme for simulating stochastic differential equations was used with an integration step of $0.1 \mathrm{ps}$ [35]. Unless explicitly indicated, the VCSEL parameters are: $\gamma_{N}=1 \mathrm{~ns}^{-1}, \gamma_{p}=$ $60 \mathrm{~ns}^{-1}, \gamma_{a}=2 \mathrm{~ns}^{-1}, \gamma_{s}=50 \mathrm{~ns}^{-1}, \kappa=300 \mathrm{~ns}^{-1}$, and $\alpha=3$. With these parameters the free-running laser emits the $y$ polarization at threshold and displays a sharp polarization switching (PS) to the orthogonal $x$ polarization at about $\mu=1.75$ [see Fig. 1(a)].

In the following we study $x$-polarized injection and $y$-polarized injection by considering a low pump current (below the PS) or a high pump current (above the PS); the noise strength, $D$, the injection strength, $E_{i n j}$, the bit time, $T$, and the detuning variation, $\Delta \omega_{\mathrm{c}}$ [Eq. (5)], are varied to find the optimal operation conditions.

While in the model the parameter $D$ represents the strength of spontaneous emission, and therefore, is a parameter that could not be varied experimentally, in practice we can consider $D$ as also representing the strength of external optical noise that is injected into the laser. This incoherent optical injection can be experimentally implemented by using an incoherent light source, such as a LED, a multimode laser or a distant laser (such that the distance is longer than the coherence length of the laser beam). In this way, a realistic low limit for varying the parameter $D$ corresponds to the typical strength of spontaneous emission in semiconductor lasers (in the range of $10^{-4}-10^{-5}$ ), while, if we assume that $D$ also represents the strength of injected incoherent light, a high limit is arbitrary. In the discussion below we will also consider very low, unrealistic values of $D$, and we will do this in order to
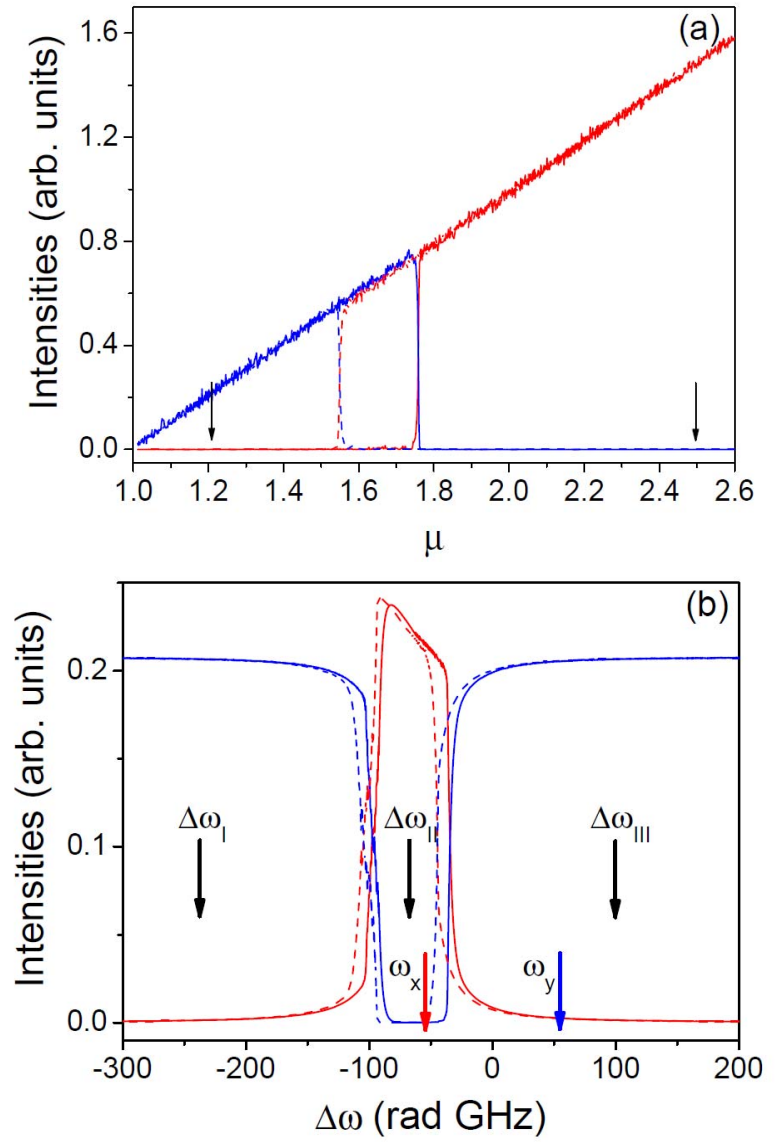

Fig. 1. (Color online) (a) Polarization-resolved intensity $\left[I_{x}\right.$ (thick, red), $I_{y}$ (thin, blue)] vs. the pump current parameter, $\mu$. The solid (dashed) lines indicate the intensities for increasing (decreasing) $\mu$. The arrows indicate the values of $\mu$ used for $x$ and $y$ polarized injection. b) Hysteresis cycle as a function of the detuning of the injected field, $\Delta \omega$, for $x$ polarized injection. The solid (dashed) lines indicate $I_{x}$ and $I_{y}$ for increasing (decreasing) $\Delta \omega$. The pump current is $\mu=1.2$, the injection strength is $E_{i n j}=0.02$, the noise strength is $D=10^{-3} \mathrm{~ns}^{-1}$ and the VCSEL parameters are as indicated in the text. The arrows indicate the three levels used to encode the logic inputs, and the angular optical frequencies of the $x$ and $y$ polarizations of the free-running VCSEL (see text for details).

demonstrate the operation principle of the stochastic logic gate, that exploits the presence of noise for robust operation (i.e., we will show that without noise or with very weak noise, the reliability of the logic gate is severely degraded).

\section{A. X-Polarized Injection}

We begin by considering a pump parameter such that the free-running VCSEL emits the $y$ polarization [i.e., $\mu<1.75$, indicated with an arrow in Fig. 1(a)] and we consider optical injection into the orthogonal $x$ polarization $\left(\kappa_{x}=\kappa, \kappa_{y}=0\right)$.

The bistable hysteresis cycle when the detuning $\Delta \omega$ varies is presented in Fig. 1(b) ( $\Delta \omega$ varies with a linear ramp in $5 \mu \mathrm{s}$ ). Figure 1(b) also indicates the three values $\Delta \omega_{\mathrm{I}}, \Delta \omega_{\text {II }}, \Delta \omega_{\text {III }}$ used for encoding the two logic inputs. These are chosen such that, under constant injection conditions, for $\Delta \omega=\Delta \omega_{\mathrm{I}}$ and for $\Delta \omega=\Delta \omega_{\text {III }}$ he VCSEL emits the $y$ polarization, and for $\Delta \omega=\Delta \omega_{\text {II }}$, the VCSEL emits the $x$ polarization ( $\Delta \omega_{\text {II }}$ is chosen in the center of the region where the $x$-polarization is on).

Figure 2 shows the time trace of the $y$ polarization, when $\Delta \omega$ varies with a fast three-level signal; Fig. 2(a) displays the 


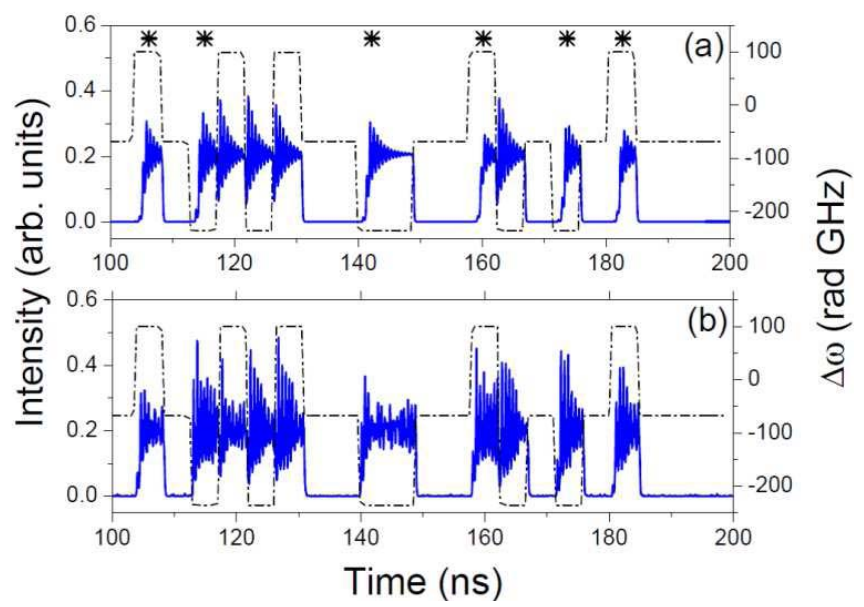

Fig. 2. (Color online) Dynamics with $x$ polarized injection (the free-running laser emits the $y$ polarization). Time trace of the intensity of the $y$ polarization (solid line), and the three-level signal that varies the detuning of the injected field (dashed dot line). The noise strength is (a) $D=10^{-6} \mathrm{~ns}^{-1}$ and (b) $10^{-3} \mathrm{~ns}^{-1}$, other parameters are $\mu=1.2, E_{\text {inj }}=0.02, T=4.5 \mathrm{~ns}$, $\Delta \omega_{\mathrm{II}}=-68 \mathrm{rad} \mathrm{GHz}, \Delta \omega_{\mathrm{c}}=168 \mathrm{rad} \mathrm{GHz}$. The $*$ indicates the wrong bits (see text for details).

behavior when the noise is weak, and Fig. 2(b), when the noise is stronger. It can be observed that with weak noise there is a considerable delay in the turn on of the $y$ polarization. In Fig. 2(a) the bits indicated with * are such that the delay in the turn on is long enough to result in a wrong bit. When the noise is stronger, Fig. 2(b), there is almost no delay in the turn on. If the noise is too strong (not shown), then both polarizations are emitted simultaneously and the logic response cannot be clearly identified.

To quantify these observations we compute the success probability $P$ using the three criteria discussed in the previous section: 90/10; 80/20; and 70/30. Figures 3(a) and 3(b) show the success probability of the OR logic gate (computed over 512 bits) as a function of the noise strength, $D$, and of the bit time, $T$, respectively. In Fig. 3(a) one can see that for the $80 / 20$ and the $70 / 30$ criteria there is a wide range of noise strengths (between $10^{-5}$ and $10^{-1} \mathrm{~ns}^{-1}$ ) where $P=1$. We remark that $T$ is of a few nanoseconds and the wide region where $P=1$ demonstrates the correct operation of the logic gate, exploiting the presence of noise. Figure 3(b) shows that with a slightly longer bit time (of about $10 \mathrm{~ns}$ ), is possible obtain $P=1$ even with the stricter $90 / 10$ criterion.

Let us now investigate the robustness of the operation and the influence of the various parameters. Figure 4 displays the success probability as a function of pump current $\mu$ and the injection strength, $E_{i n j}$ for low, intermediate and high noise. One can observe a wide region corresponding to $P=1$, which occurs for pump current values lower than the PS point.

One can also notice that an intermediate noise level (panel c) gives the wider $P=1$ region, while if the noise is too strong (panel d), then $P$ is close but not equal to 1 .

In Fig. 4 one can also notice that the parameter region where $P=1$ disappears at large values of $E_{i n j}$. This is due to the fact that, if the injected power is too strong, then only the injected polarization turns on and the VCSEL cannot operate as a logic gate. In Figs. 4(a), 4(b) and 4(c), where there is
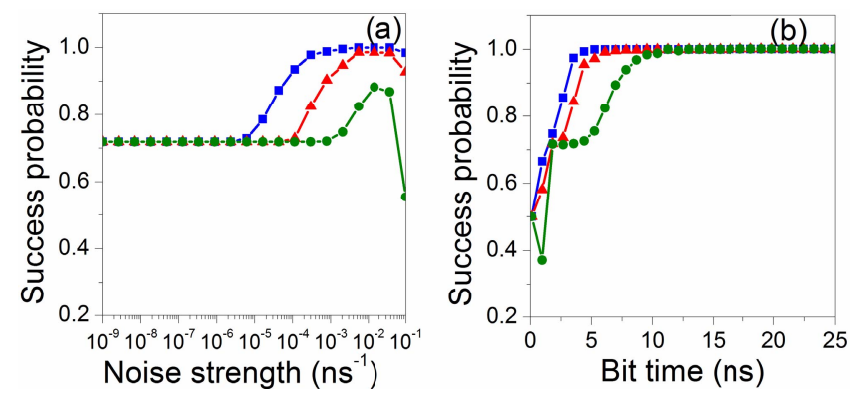

Fig. 3. (Color online) Success probability a function of the noise strength $D$ (a) and of the bit time $T$ (b) using the criteria 70/30 (squares), 80/20 (triangles), and 90/10 (circles). In (a) $T=4.5 \mathrm{ns,} \mathrm{in} \mathrm{(b)} D=10^{-3} \mathrm{~ns}^{-1}$, other parameters are as in Fig. 2.

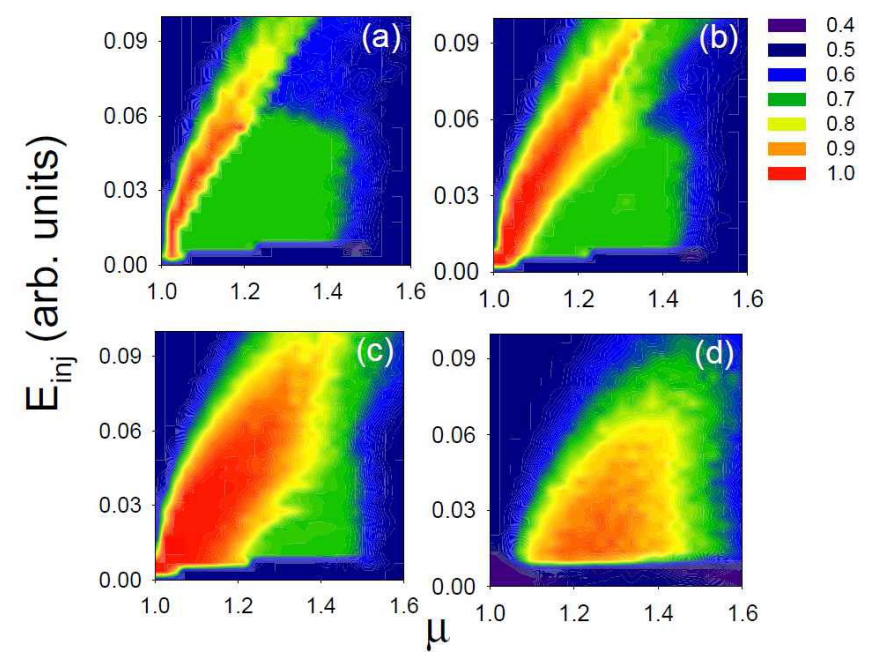

Fig. 4. (Color online) Success probability computed with 80/20 criterion, as a function of the injected field amplitude, $E_{i n j}$, and the pump current parameter, $\mu . D=10^{-9} \mathrm{~ns}^{-1}$ (a), $10^{-5} \mathrm{~ns}^{-1}$ (b), $10^{-3} \mathrm{~ns}^{-1}$ (c), and $D=10^{-1} \mathrm{~ns}^{-1}(\mathrm{~d})$; other parameters are as in Fig 2.

a clear and well defined $P=1$ region, we observe that the minimum injection strength for reliable operation increases with the pump current. This is can be due to the fact that the laser output intensity increases with the pump current and the injected field has to be stronger to produce a PS from the free-running to the orthogonal mode.

Figure 5 shows that the correct laser response does not require a fine tuning of other model parameters. As a function of the detuning variation, $\Delta \omega_{\mathrm{c}}[\mathrm{Eq} .(5)]$, there are wide parameter regions where $P=1$ varying either the noise strength $D$ (a), the pump current $\mu(\mathrm{b})$, the optical injected field $E_{i n j}(\mathrm{c})$ or the bit time $T(\mathrm{~d})$. In particular, in Fig. 5(d) one can observe that the minimum bit time such that the success probability is 1 is close to $2 \mathrm{~ns}$. This is almost 15 times faster as compared with the bit time found in Ref. [32], where the logic inputs were encoded in the modulation of the freerunning laser pump current. The faster bit time obtained here can be understood in the following terms: a switching triggered by the variation of the pump current will be slower than a switching triggered by the variation of the optical injection conditions, because the carrier density dynamics is slower than the photon density dynamics (the carrier and photon 

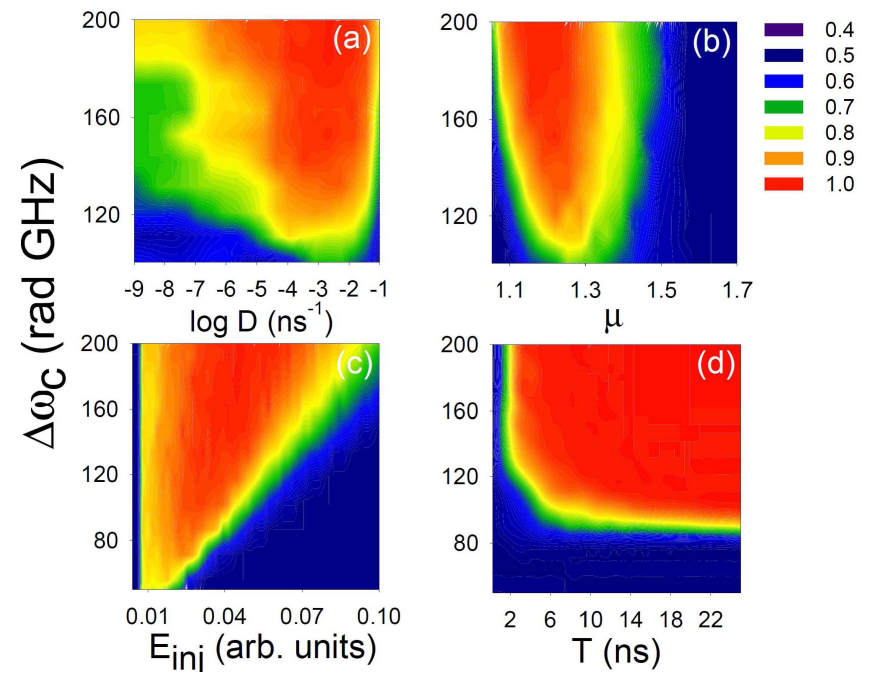

Fig. 5. (Color online) Success probability for $x$ polarized injection, as a function of the detuning variation, $\Delta \omega_{\mathrm{c}}$, and the noise strength, $D$ (a); the pump current parameter, $\mu(\mathrm{b})$; the injected field amplitude, $E_{i n j}(\mathrm{c})$ and the bit time $T$ (d). When the parameters do not vary, they are $D=10^{-3} \mathrm{~ns}^{-1}$, $E_{i n j}=0.05, \mu=1.2, T=4.5 \mathrm{~ns}$; other parameters are indicated in the text.

lifetimes being of the order of nanoseconds and picoseconds respectively). The minimum bit time found here is in fact similar to that found in Ref. [33], where the logic inputs were encoded in the strength of the injected field (see Fig. 3d of Ref. [33], where the minimum bit time is slightly above $2 \mathrm{~ns})$. While the bit times are comparable, an advantage of the present configuration with respect to that proposed in Ref. [33] is that here the encoding of the logic inputs requires using a $\mathrm{cw}$ tunable laser, which can be simpler to implement than encoding the inputs in the variation of the injection strength, as proposed in Ref. [33].

In Fig. 5(b) we observe that there is a minimum detuning variation, $\Delta \omega_{\mathrm{c}}$, for successful operation. This minimum decreases with the bit time and for long enough bit times saturates to the width of the region in Fig 1(b) where the $x$ polarization is on. The variation of the minimum $\Delta \omega_{\mathrm{c}}$ with the bit time can be understood as due to dynamical hysteresis: the width of the region where the $x$ polarization is on corresponds to that shown in Fig. 1(b) only if the control parameter $(\Delta \omega)$ varies slowly (i.e., a quasi-static variation [36]); a fast variation of $\Delta \omega$ leads to an enlargement of this region, because the bifurcations are delayed. When varying the pump current this delay was observed in Figs. 1(a) and 1(c) of Ref. [32], and we expect that this effect plays a role here when varying $\Delta \omega_{\mathrm{c}}$.

In Fig. 5(c) we also observe that there is minimum injection strength for successful operation, which is of the order of $E_{i n j}=0.005$. A minimum $E_{i n j}$ was also found in Ref. [33] (see, e.g., Figs. 4(a) and 4(b)) and, while this is not yet fully understood, we speculate that there is a minimum injection strength that is required in order to turn on the orthogonal polarization avoiding too long turn on delays that would lead to errors in the logic response.

\section{B. Y-Polarized Injection}

Let us now consider the situation in which the free-running VCSEL emits the $x$ polarization [for our parameters, this

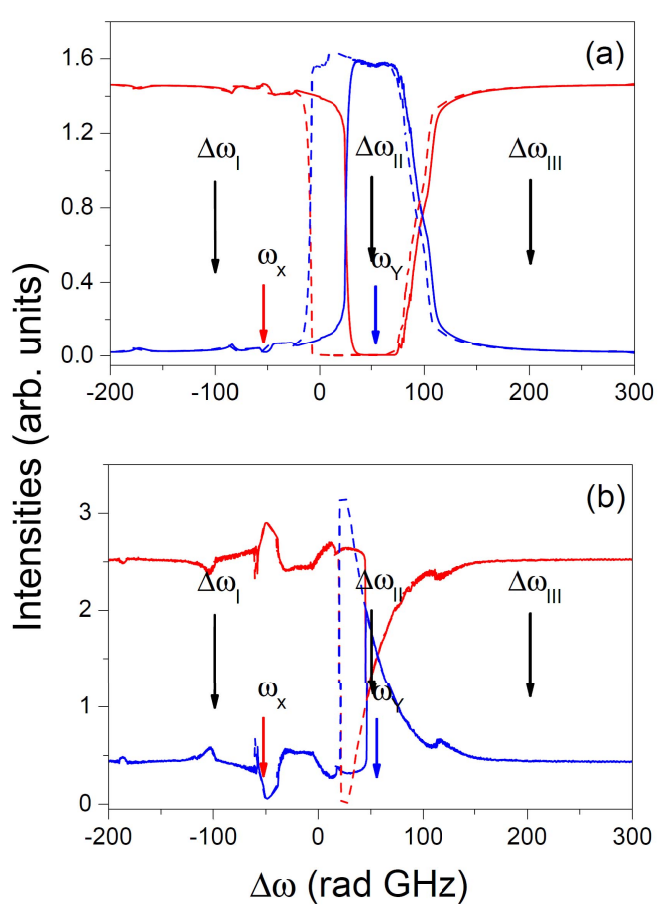

Fig. 6. (Color online) Hysteresis cycle as a function of the detuning of the injected field, for $y$ polarized injection. The solid (dashed) lines indicate the intensities $I_{x}$ and $I_{y}$ for increasing (decreasing) $\Delta \omega . E_{i n j}=0.06, D=$ $10^{-3} \mathrm{~ns}^{-1}, \mu=2.5$ (a) and $\mu=4$ (b). The VCSEL parameters are as indicated in the text. The arrows indicate the three levels used to encode the logic inputs and the angular optical frequencies of the $x$ and $y$ polarizations of the free-running VCSEL (see text for details).

occurs for $\mu>1.75$, see Fig. 1(a)] and we consider optical injection into the $y$ polarization $\left(\kappa_{y}=\kappa, \kappa_{x}=0\right)$. The bistable hysteresis cycle for two values of $\mu$ is presented in Fig. 6, where the detuning $\Delta \omega$ varies with a linear ramp in $5 \mu \mathrm{s}$. The arrows indicate the values of $\Delta \omega_{\mathrm{I}}, \Delta \omega_{\mathrm{II}}$ and $\Delta \omega_{\text {III }}$ used to encode the logic inputs. Now $\Delta \omega_{\text {II }}$ is in the center of the region where the $y$ polarization turns on.

As indicated in Table I, for $y$ polarized injection, when $\Delta \omega=\Delta \omega_{\mathrm{I}}$ and $\Delta \omega=\Delta \omega_{\text {III }}$ the VCSEL gives the correct logic output if it emits the $x$ polarization, and when $\Delta \omega=\Delta \omega_{\text {II }}$, it gives the correct logic output if it emits the $y$ polarization. To compute the success probability we now calculate the intensity emitted in the injected $y$ polarization, and apply the same three criteria discussed at the end of section III: in levels $\Delta \omega=\Delta \omega_{\mathrm{I}}$ and $\Delta \omega=\Delta \omega_{\mathrm{III}}, x$ is the correct polarization and therefore, the light emitted with $y$ polarization has to be lower than a certain percentage of the total emitted light $(10 \%$, or $20 \%$ or $30 \%)$, while in level $\Delta \omega=\Delta \omega_{\text {II }}, y$ is the correct polarization and the light emitted with $y$ polarization has to be larger than a certain percentage of the total emitted light (90\%, or $80 \%$ or $70 \%$ ).

Figure 7 shows the time trace of the $x$ polarization for two noise levels, when $\Delta \omega$ varies with a three-level signal. One can notice that, if the noise is too weak [Fig. 7(a)], there are wrong bits, indicated with *, which are due to the fact that the $x$ polarization delays to turn on, or does not turn on completely when $\Delta \omega=\Delta \omega_{\text {III }}$ or $\Delta \omega=\Delta \omega_{\text {I. }}$. These wrong bits can be corrected with adequate noise strength [Fig. 7(b)]. 


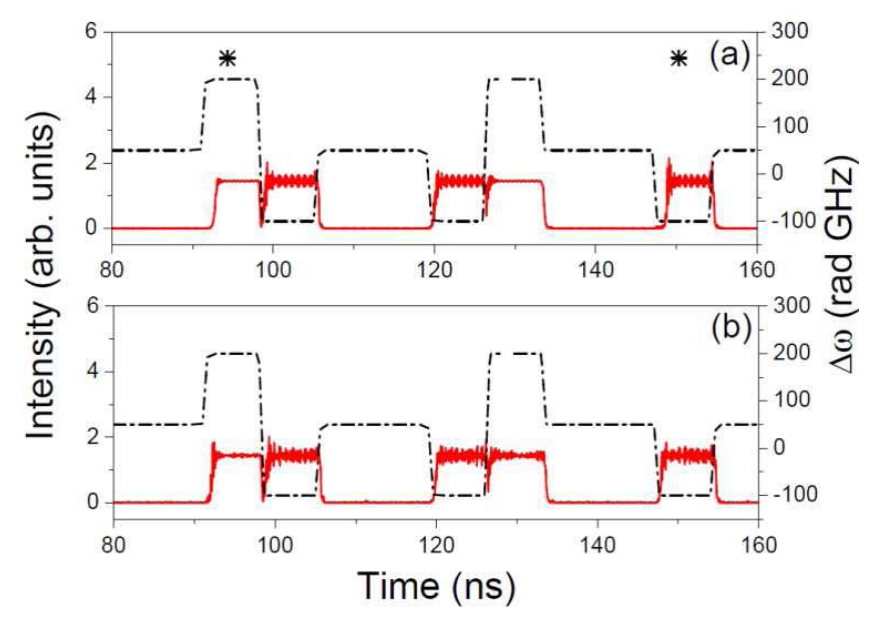

Fig. 7. (Color online) Dynamics with $y$ polarized injection (the free-running laser emits the $x$ polarization). Time trace of the intensity of the $x$ polarization (solid line), and the three-level signal that varies the detuning of the injected field (dashed dot line). The noise strength is (a) $D=10^{-5} \mathrm{~ns}^{-1}$, (b) $D=$ $10^{-2} \mathrm{~ns}^{-1}$, other parameters are $\mu=2.5, E_{i n j}=0.06, T=7 \mathrm{~ns}, \Delta \omega_{\mathrm{II}}=$ $50 \mathrm{rad} \mathrm{GHz}, \Delta \omega_{\mathrm{c}}=150 \mathrm{rad} \mathrm{GHz}$. The $*$ indicates the wrong bits (see text for details).
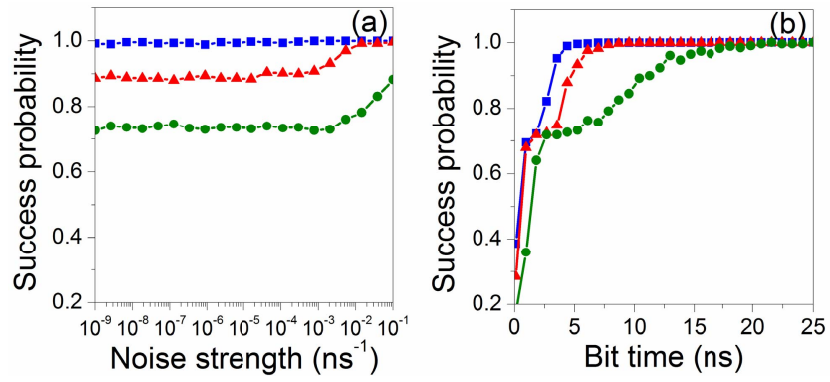

Fig. 8. (Color online) Success probability for $y$-polarized injection, as function of the noise strength (a) and bit time (b) using the criteria of 70/30 (squares), 80/20 (triangles), and 90/10 (circles). In (a) $T=7 \mathrm{~ns}$, in (b) $D=10^{-2} \mathrm{~ns}^{-1}$; other parameters are as in Fig 6 .

Figure 8 shows the success probability as a function of the noise strength [Fig. 8(a)] and of the bit time [Fig. 8(b)]. One can observe in Fig. 8(a) that the success probability is rather insensitive to the noise level, at least for noise levels below $D=10^{-1} \mathrm{~ns}^{-1}$. This lack of sensitivity can be due to the fact that the pump current is higher than for $x$ injection (and thus, noise can be less relevant), or it can be due to different injection conditions. Figure 8(b) shows that with the less restrictive criteria (70/30 and 80/20) and $D=10^{-3} \mathrm{~ns}^{-1}$, it is possible obtain $P=1$ for bit times of $4 \mathrm{~ns}$ and 7 ns respectively, while for the more restrictive criteria 90/10, longer bit-times are required (above $20 \mathrm{~ns}$ ).

Figure 9 shows the success probability for $y$-polarized injection in the $E_{i n j}-\mu$ plane for low, intermediate and high noise strengths. There are wide regions of parameters where, with an adequate level of noise, the VCSEL operates as a logic gate. However, within the region where the success probability is close to one, we can observe an island where success probability decreases significantly [see Figs. 9(a), 9(b), and 9 (c)]. In the bistable hysteresis cycle, for these $E_{i n j}-\mu$ values we observe the excitation of the $x$ polarized mode in the positive detuning range in between the two PS points. This excitation of the $x$ polarized mode coincides with a

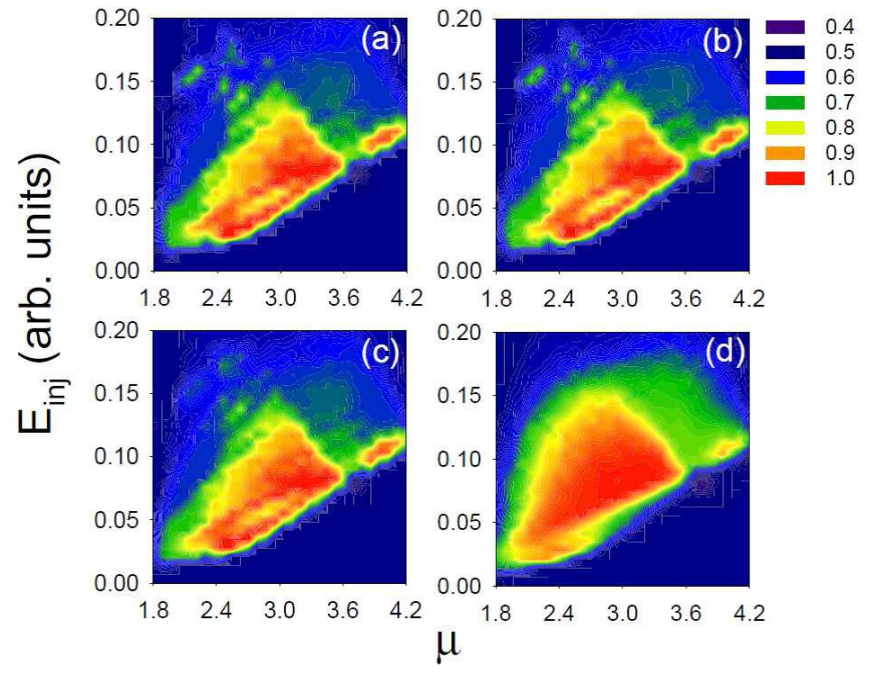

Fig. 9. (Color online) Success probability whit $y$ polarized injection as a function of the pump current parameter, $\mu$ for noise strength: $D=10^{-9} \mathrm{~ns}^{-1}$ (a), $10^{-5} \mathrm{~ns}^{-1}$ (b), $10^{-3} \mathrm{~ns}^{-1}$ (c), and $10^{-1} \mathrm{~ns}^{-1}$ (d). $T=5 \mathrm{~ns}$ and $\Delta \omega_{\mathrm{c}}=150 \mathrm{rad} \mathrm{GHz}$, other parameter as in Fig. 7 .

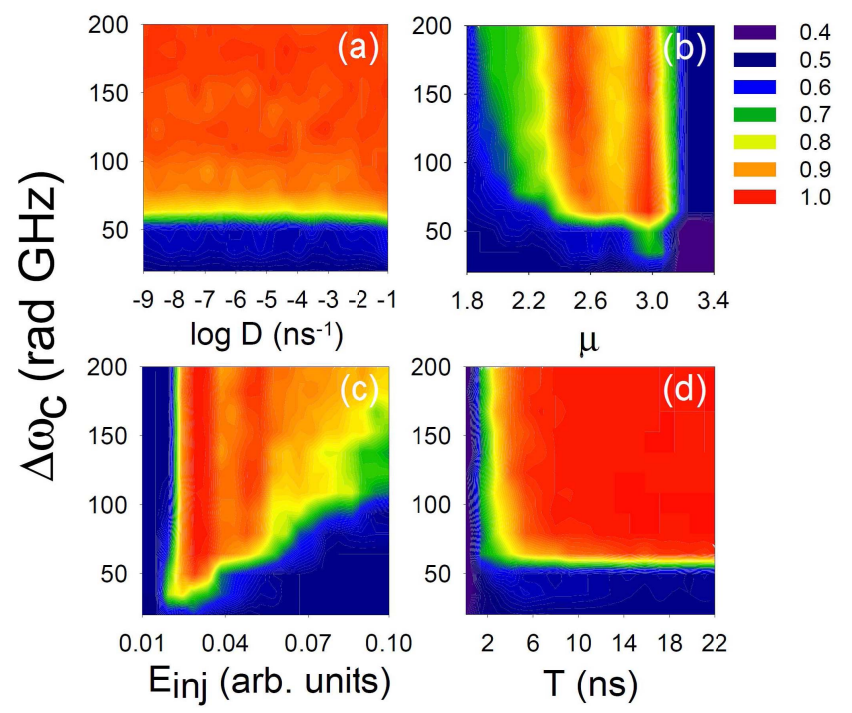

Fig. 10. (Color online) As Fig. 5 but with $y$ polarized injection. When the parameters do not vary they are: $\mu=2.5, E_{i n j}=0.05, T=5 \mathrm{~ns}$, and $D=10^{-2} \mathrm{~ns}^{-1}$.

decrease of the averaged intensity of the $y$ polarized mode, as discussed in [22]. In this positive detuning range the two modes strongly compete and do not allow decoding the logic output response.

In Fig. 9 we also observe that there is a minimum $E_{i n j}$, which tends to increase with the pump current (as in Fig. 4 for $x$ polarized injection). Also in analogy with $x$ injection, where the maximum pump current for successful operation was the PS point of the free-running laser, now the minimum value of the pump current for successful operation is the PS point.

Figure 10 shows that also for $y$-polarized injection the correct operation does not require a fine tuning of the VCSEL parameters: $P$ is equal to 1 in a wide parameter region, varying either the noise strength $D$ (a), the pump current $\mu$ (b), the injection strength, $E_{i n j}(\mathrm{c})$ or the bit time, $T$ (d). 
In Figs. 9 and 10 one can observe that the $P=1$ region is lost when the pump current is too high. This occurs because the two modes are simultaneously on, as can be observed in Fig. 6(b).

In Fig. 10(c) we observe that there is a minimum injection strength required for successful operation, which is higher than that for $x$ injection [compare with Figs. 5(c)], which can be due to the fact that the output power of the free-running VCSEL is higher (as the pump parameter is higher), and therefore, higher (minimum) injection power is required for successful operation. In Fig. 10(d) the minimum bit time is close to $2 \mathrm{~ns}$, and there is a minimum detuning variation, $\Delta \omega_{\mathrm{c}}$, which decreases with the bit time and saturates to the width of the injection-locking region, as seen in the case of $x$ injection.

\section{CONCLUSION}

The dynamics of a VCSEL with orthogonal optical injection was studied numerically. We demonstrated that the interplay of injection, polarization bistability and noise allows for implementing a reliable stochastic logic gate. Specifically, we propose an all-optical logic gate, with the two logic inputs encoded in the detuning of the injected light, and the response decoded from the polarization state of the emitted light.

We presented a detailed analysis in terms of the laser parameters and of the injection parameters. We compared the performance of two orthogonal injection schemes: in the low-frequency, $x$ polarization (for pump currents below the polarization switching point of the free-running laser) and in the high-frequency, $y$ polarization (for pump currents above the switching point of the free-running laser). We showed that this configuration works more than ten times faster than the implementation in Ref. [32] and is as fast as that in Ref. [33], while it offers the advantage of being simpler, operating at constant injection power.

We also presented a detailed comparison of the operation with $x$ polarized or with $y$ polarized injection. The noise influence is most relevant in the case of $x$ polarized injection, perhaps because the device operates below the polarization switching point and closer to the threshold. The regions where the success probability is close to one show the same dependency on the parameters for both, $x$ or $y$ polarized injection. In both cases, for large detuning variation is possible to obtain a success probability equal to one with bit times of the order of $2 \mathrm{~ns}$.

\section{REFERENCES}

[1] H. Kawaguchi, "Polarization-bistable vertical-cavity surface-emitting lasers: Application for optical bit memory," Opt. Electron. Rev., vol. 17, no. 4, pp. 265-274, Dec. 2009.

[2] T. Katayama, T. Ooi, and H. Kawaguchi, "Experimental demonstration of multi-bit optical buffer memory using $1.55-\mu \mathrm{m}$ polarization bistable vertical-cavity surface-emitting lasers," IEEE J. Quantum Electron., vol. 45, no. 11, pp. 1495-1504, Nov. 2009.

[3] A. Larsson, "Advances in VCSELs for communication and sensing," IEEE J. Sel. Topic Quantum Electron., vol. 17, no. 6, pp. 1552-1567, Nov./Dec. 2011

[4] Z. G. Pan, S. Jiang, M. Dagenais, R. A. Morgan, K. Kojima, M. T. Asom, and R. E. Leibenguth, "Optical injection induced polarization bistability in vertical-cavity surface-emitting lasers," Appl. Phys. Lett., vol. 63 , no. 22, pp. 2999-3001, Sep. 1993.
[5] S. Wieczorek, W. W. Chow, L. Chrostowski, and C. J. Chang-Hasnain, "Improved semiconductor-laser dynamics from induced population pulsation," IEEE J. Quantum Electron., vol. 42, no. 6, pp. 552-562, Jun. 2006.

[6] L. Chrostowski, B. Faraji, W. Hofmann, M. C. Amann, S. Wieczorek, and W. W. Chow, " $40 \mathrm{GHz}$ bandwidth and $64 \mathrm{GHz}$ resonance frequency in injection-locked $1.55 \mu \mathrm{m}$ VCSELs," IEEE J. Sel. Top. Quantum Electron., vol. 13, no. 5, pp. 1200-1208, Sep./Oct. 2007.

[7] A. Homayounfar and M. J. Adams, "Analysis of SFM dynamics in solitary and optically-injected VCSELs," Opt. Exp., vol. 15, no. 17, pp. 10504-10519, Aug. 2007.

[8] V. Kovanis, A. Gavrielides, and J. A. C. Gallas, "Labyrinth bifurcations in optically injected diode lasers," Eur. Phys. J. D, vol. 58, no. 2, pp. 181-186, Mar. 2010.

[9] C. Bonatto, M. Feyereisen, S. Barland, M. Giudici, C. Masoller, J. R. Rios Leite, and J. R. Tredicce, "Deterministic optical rogue waves," Phys. Rev. Lett., vol. 107, no. 5, pp. 053901-1-053901-5, Jul. 2011.

[10] M. San-Miguel, "Polarization properties of vertical cavity surface emitting lasers," in Semiconductor Quantum Optoelectronics, A. Miller, M. Ebrahimzadeh, and D. M. Finlayson, Eds. Bristol, U.K.: Inst. Phys., 1999, pp. 339-366

[11] J. Y. Law, G. H. M. van Tartwijk, and G. P. Agrawal, "Effects of transverse mode competition on the injection dynamics of verticalcavity surface-emitting lasers," Quantum Semiclass. Opt., vol. 9, no. 5, pp. 737-747, Oct. 1997.

[12] M. S. Torre, C. Masoller, and K. A. Shore, "Numerical study of optical injection dynamics of vertical-cavity surface-emitting lasers," IEEE J. Quantum Electron., vol. 40, no. 1, pp. 25-30, Jan. 2004.

[13] Y. Onishi, F. Koyama, N. Nishiyama, C. Caneau, and C. E. Zah, "Nonlinear optical input-output characteristics of $1.55 \mu \mathrm{m}$ injectionlocked vertical-cavity surface-emitting lasers," Appl. Phys. Lett., vol. 84, no. 17, pp. 3247-3249, Apr. 2004.

[14] M. S. Torre, A. Valle, and L. Pesquera, "Polarization and transverse mode behavior of VCSELs under optical injection," Opt. Quantum Electron., vol. 38, nos. 4-6, pp. 445-465, Mar. 2006.

[15] M. S. Torre, A. Valle, and L. Pesquera, "Transverse mode selection in vertical-cavity surface-emitting lasers with optical injected signal," IEEE J. Quantum Electron., vol. 46, no. 1, pp. 105-111, Jan. 2010.

[16] M. Salvide, M. S. Torre, A. Valle, and L. Pesquera, "Transverse mode selection and bistability in vertical-cavity surface-emitting lasers induced by parallel polarized optical injection," IEEE J. Quantum Electron., vol. 47, no. 5, pp. 723-730, May 2011.

[17] Y. Hong, K. A. Shore, A. Larsson, M. Ghisoni, and J. Halonen, "Pure frequency-polarisation bistability in vertical-cavity surfaceemitting lasers subject to optical injection," Electron. Lett., vol. 36, no. 24, pp. 2019-2020, Nov. 2000.

[18] B. S. Ryvkin, K. Panajotov, E. A. Avrutin, I. Veretennicoff, and $\mathrm{H}$. Thienpont, "Optical-injection-induced polarization switching in polarization-bistable vertical-cavity surface-emitting lasers," J. Appl. Phys., vol. 96, no. 11, pp. 6002-6007, Aug. 2004.

[19] M. Sciamanna and K. Panajotov, "Route to polarization switching induced by optical-injection in vertical-cavity surface-emitting lasers," Phys. Rev. A, vol. 73, no. 2, pp. 023811-1-023811-17, Feb. 2006.

[20] J. Buesa, I. Gatare, K. Panajotov, H. Thienpont, and M. Sciamanna, "Mapping of the dynamics induced by orthogonal optical injection in vertical-cavity surface-emitting laser," IEEE J. Quantum Electron., vol. 42, no. 2, pp. 198-207, Feb. 2006.

[21] I. Gatare, J. Buesa, H. Thienpont, K. Panajotov, and M. Sciamanna, "Polarization switching bistability and dynamics in vertical-cavity surface-emitting lasers under orthogonal optical injection," Opt. Quantum Electron., vol. 38, nos. 4-6, pp. 429-443, Mar. 2006.

[22] I. Gatare, K. Panajotov, and M. Sciamanna, "Frequency-induced polarization bistability in vertical-cavity surface-emitting lasers with orthogonal optical injection," Phys Rev. A, vol. 75, pp. 023804-1-023804-7, Feb. 2007.

[23] A. Valle, I. Gatare, K. Panajotov, and M. Sciamanna, "Transverse mode switching and locking in vertical-cavity surface-emitting lasers subject to orthogonal optical injection," IEEE J. Quantum Electron., vol. 43, no. 4, pp. 322-333, Apr. 2007.

[24] I. Gatare, M. Sciamanna, M. Nizette, and K. Panajotov, "Bifurcation to polarization switching and locking in vertical-cavity surfaceemitting lasers with optical injection," Phys. Rev. A, vol. 76, no. 3, pp. 031803-1-031803-4, Sep. 2007. 
[25] A. Hurtado, I. D. Henning, and M. J. Adams, "Two-wavelength switching with a $1550 \mathrm{~nm}$ VCSEL under single orthogonal optical injection," IEEE J. Sel. Topics Quantum Electron, vol. 14, no. 3, pp. 911-917, May/Jun. 2008.

[26] A. Valle, M. Gomez-Molina, and L. Pesquera, "Polarization bistability in $1550 \mathrm{~nm}$ wavelength single-mode vertical-cavity surface-emitting lasers subject to orthogonal optical injection," IEEE J. Sel. Topics Quantum Electron., vol. 14, no. 3, pp. 895-902, May/Jun. 2008.

[27] A. Hurtado, I. D. Henning, and M. J. Adams, "Different forms of wavelength polarization switching and bistability in a $1.55 \mathrm{~m}$ vertical.-cavity surface-emitting laser under orthogonally polarized optical injection," Opt. Lett., vol. 34, no. 3, pp. 365-367, Feb. 2009.

[28] M. S. Torre, A. Quirce, A. Valle, and L. Pesquera, "Wavelength-induced polarization bistability in $1550 \mathrm{~nm}$ VCSELs subject to orthogonal optical injection," J. Opt. Soc. Amer. B, vol. 27, no. 12, pp. 2542-2548, Dec. 2010.

[29] M. S. Torre, A. Hurtado, A. Quirce, A. Valle, L. Pesquera, and M. J. Adams, "Polarization switching in long-wavelength verticalcavity surface-emitting lasers subject to orthogonal optical injection," IEEE J. Quantum Electron., vol. 47, no. 1, pp. 92-99, Jan. 2011.

[30] K. Murali, S. Shina, W. L. Ditto, and A. R. Bulsara, "Reliable logic circuit elements that exploit nonlinearity in the presence of a noise floor,' Phys. Rev. Lett., vol. 102, no. 10, pp. 104101-1-104101-4, Mar. 2009.

[31] K. Murali, I. Rajamohamed, S. Shina, W. L. Ditto, and A. R. Bulsara, "Realization of reliable and flexible logic gates using noisy nonlinear circuits," Appl. Phys. Lett., vol. 95, no. 19, pp. 194102-1-194102-3, Nov. 2009.

[32] J. Zamora-Munt, and C. Masoller, "Numerical implementation of a VCSEL-based stochastic logic gate via polarization bistability," Opt. Exp., vol. 18, no. 16, pp. 16418-16429, Aug. 2010.

[33] S. Perrone, R. Vilaseca, and C. Masoller, "Stochastic logic gate that exploits noise and polarization bistability in an optically injected VCSEL," Opt. Exp., vol. 20, no. 20, pp. 22692-22699, Sep. 2012.

[34] J. M. Martín-Regalado, F. Prati, M. San Miguel, and N. B. Abraham, "Polarization properties of vertical cavity surface-emitting lasers," IEEE J. Quantum Electron., vol. 33, no. 5, pp. 765-783, May 1997.
[35] P. E. Kloeden and E. Platen, Numerical Solution of Stochastic Differential Equations, vol. 23, New York, NY, USA: Springer-Verlag, 1992.

[36] C. Masoller, M. S. Torre, and P. Mandel, "Influence of the injection current sweep rate on the polarization switching of vertical cavity surface-emitting lasers," J. Appl. Phys., vol. 99, pp. 026108-1-026108-3 Jan. 2006.

Matias F. Salvide received the Licenciado en Física (M.Sc.) degree from Universidad Nacional del Centro de la Provincia de Buenos Aires, Buenos Aires, Argentina, in 2012, where he is currently pursuing the Ph.D. degree in physics. His current research interests include dynamics of vertical-cavity surface-emitting lasers and optical injection effects in semiconductor lasers.

Cristina Masoller received the M.Sc. degree in physics from Universidad de la Republica, Montevideo, Uruguay, in 1991, and the Ph.D. degree in physics from Bryn Mawr College, Bryn Mawr, PA, USA, in 1999. Since 2004, she has been with Universitat Politecnica de Catalunya, Catalunya, Spain. Her current research interests include nonlinear dynamics of semiconductor lasers, synchronization and stochastic phenomena, and time-delayed complex systems.

Maria Susana Torre received the Licenciada en Física (M.Sc.) and Ph.D. degrees from Universidad Nacional del Centro de la Provincia de Buenos Aires (UNCPBA), Buenos Aires, Argentina. Since 1988, she has been a member of the Physics Institute "Arroyo Seco," IFAS, UE CIFICEN. She is currently a Research Professor with the Facultad de Ciencias Exactas of the UNCPBA and Independent Researcher National Council of Scientific and Technical Investigations. Her current research interests include modeling and dynamics of VCSEL, dynamics of semiconductor lasers with external optical feedback, and diffusion effects in semiconductor lasers, optical bistability. She is currently working on the modeling of nonlinear properties of semiconductor lasers and their applications to optical communication systems. 